\title{
A study on new method of noninvasive esophageal venous pressure measurement based on the airflow and laser detection technology
}

\author{
Chenghuan $\mathrm{Hu}_{\mathrm{b}}^{\mathrm{a}}$, Feizhou Huang ${ }^{\mathrm{a}}$, Rui Zhang ${ }^{\mathrm{a},{ }^{*}}$, Shaihong Zhu ${ }^{\mathrm{a}}$, Wanpin Nie ${ }^{\mathrm{a}}$, Xunyang Liu ${ }^{\mathrm{a}}$, \\ Yinglong Liu and Peng $\mathrm{Li}^{\mathrm{c}}$ \\ ${ }^{a}$ Department of General Surgery, The Third Xiangya Hospital of Central South University, 138 \\ Tongzipo Road, Yuelu District, Changsha, 410013, China \\ ${ }^{b}$ School of Information Science and Engineering, Central South University, 22 Shaoshan South Road, \\ Tianxin District, Changsha, 410083, China \\ ${ }^{c}$ School of Traffic and Transportation Engineering, Central South University, 22 Shaoshan South \\ Road, Tianxin District, Changsha, 410083, China
}

\begin{abstract}
Using optics combined with automatic control and computer real-time image detection technology, a novel noninvasive method of noncontact pressure manometry was developed based on the airflow and laser detection technology in this study. The new esophageal venous pressure measurement system was tested in-vitro experiments. A stable and adjustable pulse stream was produced from a self-developed pump and a laser emitting apparatus could generate optical signals which can be captured by image acquisition and analysis system program. A synchronization system simultaneous measured the changes of air pressure and the deformation of the vein wall to capture the vascular deformation while simultaneously record the current pressure value. The results of this study indicated that the pressure values tested by the new method have good correlation with the actual pressure value in animal experiments. The new method of noninvasive pressure measurement based on the airflow and laser detection technology is accurate, feasible, repeatable and has a good application prospects.
\end{abstract}

Keywords: Airflow, laser, noninvasive, pressure measurement

\section{Introduction}

Esophageal varices (EV) are one of the common complications for cirrhotic patients. Due to portal hypertension, collateral circulation develops in the lower esophagus and thus the small blood vessels in intramural esophagus become distended, appearing as varicosities [1]. The cardinal symptom is

\footnotetext{
* Address for correspondence: Rui Zhang, Department of General Surgery, The Third Xiangya Hospital of Central South University, 138 Tongzipo Road, Yuelu District, Changsha, 410013, China. Tel.: 86073188618830; Fax: 86073188618030; E-mail: rui.zhang@csu.edu.cn.
} 
esophageal variceal bleeding, and approximately $15 \% \sim 20 \%$ of the patients suffering from bleeding will die in six weeks [2]. Therefore, for high-risk population of esophageal variceal bleeding in cirrhotic patients, how to effectively and timely make diagnoses and thus predict the probability of bleeding in advance would be particularly important. Since the 1950s, numerous studies have demonstrated that too high esophageal variceal pressure could directly cause esophageal variceal bleeding [3-5]. By means of endoscopy, there are two methods of measuring esophageal variceal pressure at present; specifically, one is to measure the intravenous pressure through variceal puncture and the other is performed non-invasively outside the vein [6]. The former method was firstly proposed by Palmer in 1951 and generally recognized as a standard measurement method [7]. The variceal pressure can be directly measured by a fine needle puncture; however, this method can easily cause bacterial infection and cannot provide repeated measurements. More seriously, approximately a third of patients may suffer from hemorrhage. This method now is thus seldom adopted. The non-invasive measurement (also known as minimally invasive measurement) which is conducted outside the vein is a mainstream method for esophageal variceal pressure measurement. In 1982, based on the principle of respiratory pressure measurement, Mosimann, a Swiss researcher, firstly put forward an endoscopic varieal pressure measurement method [8]. Afterwards, many researchers have been working on improving this technique and already achieved a lot of fruitful results. Our team has been committed to non-invasive measurement of esophageal variceal pressure and the prediction of esophageal variceal bleeding. We have independently developed an endoscopic manometer for detecting esophageal variceal pressure. The in-vitro experiments, animal tests and clinical tests all indicated that the measurement results using this endoscopic manometry are in good consistent with the standard results measured by variceal puncture [9]. In 1987, also based on the principle of respiratory pressure measurement, a Swiss research group led by Gertsch developed an endoscopic balloon technique using the principle of cuff pressure measurement [10]. Currently, with the introduction of computer video processing technique, this endoscopic balloon technique has become more and more objective and accurate [11-13]. However, these two non-invasive methods should be in direct contact with esophageal veins, i.e., the iatrogenic esophageal variceal bleeding may be inevitable during measurement process. Secondly, using these two methods, the changes in tension of varicose vein walls are neglected, and the effects of deglutition, the peristaltic waves in esophagus and cardiac movements on measurement results cannot be eliminated. Therefore, the measurement results may be not very accurate. Besides, using balloon manometry, we can only observe the inner wall of balloon through videos or images. In practical applications, several problems such as light reflection and inconsistent absorption spectra will appear even if the balloon is transparent, which will be not convenient for doctors to make intuitive diagnoses.

Aiming at overcoming the above-described shortcomings, we have performed a lot of studies on a non-contact manometry which combines airflow and laser detection technology, and proposed a novel non-invasive measuring system for esophageal variceal pressure. Based on our previous studies on endoscopic manometer, this non-contact technique takes advantage of optical principles, automatic control technology and computer real-time image detection technique, and thus can provide the results with favorable accuracy and excellent repeatability in a more secure way [14]. Using this method, the active airflow with continuously varying pressures rather than balloon is adopted to compress the veins to be measured, and the deformations in esophageal veins can be detected by means of fiber laser. Accordingly, the pressure in esophageal veins can be accurately measured. This non-contact manometry, which has broken through the limits of traditional esophageal variceal pressure measurements, can meet a series of requirements in clinical applications, namely, safety (since it is non-invasive and non-contact), high accuracy, multi-point, repeated and rapid detections. Therefore, 
this manometry can effectively predict esophageal variceal bleeding.

\section{Materials and methods}

\subsection{Principles and components of the system}

In the system, the airflow is generated by an air pump, and then transported to the gas pipeline at a certain pressure and flow velocity. Through the biopsy channel for gastroscope, the gas pipeline gets close to the esophageal vein. The surface of the varicose veins is then vertically impacted by the airflow from the pipeline which is located at a certain distance away, and the impact pressure of airflow increases gradually. Within the detection area, the gravity has little effect and thus can be ignored. Along the direction which is perpendicular to the vascular wall, the impact force of airflow, the pressure in the vessel and the tension of vessel all exist. When the airflow pressure impacted on the surface of vascular wall approximates to the internal pressure of vessel, this surface is deformed. In the instant when the vascular wall is flattened, the tension vector is parallel to the vascular wall, and no force is applied vertically on any kind of vessels. According to the principle of mechanical equilibrium, the airflow impact force is equal to the pressure of vein. During the whole measurement process, the image processing software and the information of airflow system are integrated to be synchronous in the system software, so as to achieve the motion capture of the changes in vascular wall. Furthermore, the airflow pressure at the initial position of gas pipeline can be recorded, and therefore the impact pressure of airflow on the vessel can be calculated.

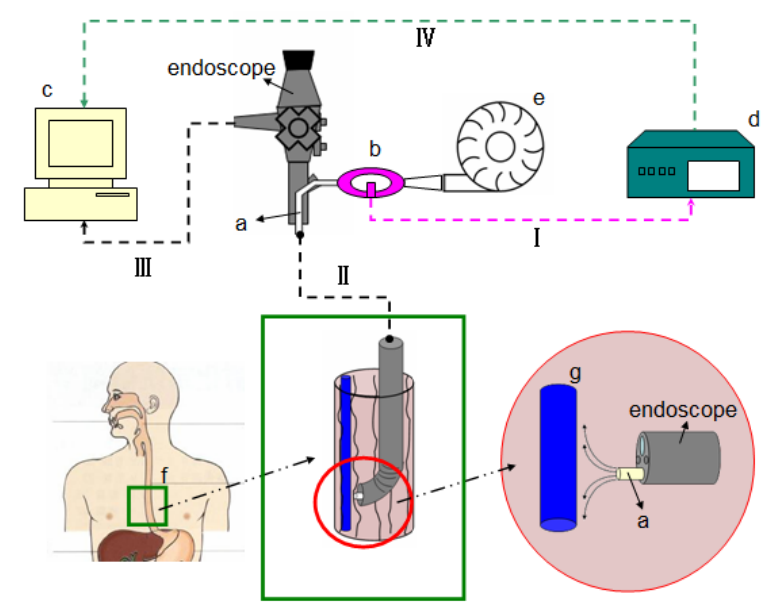

Fig. 1. Illustration of the novel non-invasive esophageal variceal pressure measuring system.

Note: a. gas pipeline; b. airflow pressure sensor; c. host system; d. pressure signal analyzer; e. adjustable air pump; f. human esophagus; g. esophageal varicose vein. The meaning of each line: I-the data are transmitted to the recorder through the pressure sensor; II-gastroscope channel; III-the image signals from the gastroscope are transmitted to the host system; and IV-the pressure signals are transmitted to the host system. The working principle of the system will be described in detail as follows. Using the adjustable air pump (e), the airflow is generated and transmitted to the gas pipeline (a). The gas pipeline arrives at the esophageal varicose vein through gastroscope channel (along the Line II). The surface of the varicose veins (g) is vertically impacted by the airflow from the pipeline which is located at a certain distance away. During the measurement process, the image signals collected by the gastroscope are then transmitted to the host system (along the Line III), and the host system judges whether the vessel is deformed or not. Simultaneously, a pressure sensor (b) is installed to record the airflow pressure at the inlet. Then the pressure signals are transmitted back to the analyzer (along the Line I). At the moment when the vessel is judged to be deformed by the host system, the pressure value recorded by the analyzer is transmitted to the host system. Finally, the data are analyzed and calculated in the host system and pressure value inside the vessel can be acquired. 
As illustrated in Figure 1, the system consists of the existing gastroscope, airflow probe system, laser distance measuring sensor, image acquisition and analysis system and central data synchronous processing system. The gastroscope can be used for direct diagnoses of esophageal varices. In the present system, the gastroscope provides the access channel for airflow and fiber optics bundle, which can thus be adjusted and operated in vitro. The airflow probe system is composed of air pump, air container, airflow pressure control valve and output pipeline, which can provide the airflow beam with continuously varying pressures and impose impacts on the vascular wall. Meanwhile, according to the functional relation of airflow pressure at different positions, the airflow pressure on the surface of vein can be calculated. With the adoption of invisible laser, the distance measuring light source can improve the detection precision without any influence on the illumination of gastroscope. The fiber optics bundle (the diameter of microobjective is less than $1 \mathrm{~mm}$ ) is fixed inside the output pipeline. The fiber laser is used 650nm FG530A standard visual laser source, a cylindrical mirror is used to form a fan shaped laser beam. The mechanism is used to measure the distance with laser by a triangulation method. Using the polarizing beam-splitting system and laser filter, the effects induced by the light reflected by film mirror and the lighting source in gastroscope can be eliminated. TMS320DM642 platform provides real-time and complex mathematical processing on two channels of video image flows, i.e., the video detection can be achieved. In image acquisition and analysis system, the interfacial design and multithread programming are conducted under the framework of Microsoft Foundation Classes (MFC), and the system flow control is achieved. The image data are analyzed using the methods described in Open Source Computer Vision Library (OpenCV), so as to identify whether the airflow is vertically applied on the surface of vein and capture the image at the moment when the vein is flattened. The developed system use open multimedia application platform (OMAP-4430, 1GHz, dual-core Cortex-A9 processor), Android operating system and some related driver and control modules. By combining with the video acquisition and image analysis system in the gastroscope, the central data synchronous processing host system is constructed. During the measurement, the host system can simultaneously retrieve the dynamic image signals in image acquisition and analysis system and the measurement data of pressure sensor in airflow probe system, and adjust the time shafts of two signals to be synchronous.

\subsection{In-vitro experiments}

The in-vitro experiments were conducted by strictly following the guidelines for the use of laboratory animals formulated by the Ethic Committee in Central South University. In the experiments, the selected six rabbits were provided by Laboratory Animal Center, the Third Xiangya Hospital of Central South University, and the weights approximately range from 2.2 to $3.0 \mathrm{~kg}$. The venous indwelling needles were embedded in the rabbits' auricular veins. For each rabbit, $2 \mathrm{mg} / \mathrm{kg}$ ethocaine were injected. After anesthesia, the rabbits were placed on the dissecting table, and disinfection and skin preparation were conducted on their bellies. For each rabbit, after the median abdominal incision was made, the subcutaneous fat and muscle layer were separated using blunt dissection. The bowels were then moved to the left side of the abdomen, and the vena cava was exposed. One branch of vena cava was selected for needle puncture measurement of variceal pressure. The indwelling catheter for vein puncture was placed and connected to BL-410 Biological Functional Experiment System (as shown in Figures 2 and 3). At the position close to the heart along the exposed vena cava, we made a line knot around the vena cava using the surgical lines. The line knot can affect the haemal circumfluence in the vena cava and the internal pressure can also be varied by controlling the tightness degree of the knot. The probe of the developed non-invasive measuring system was then placed right 


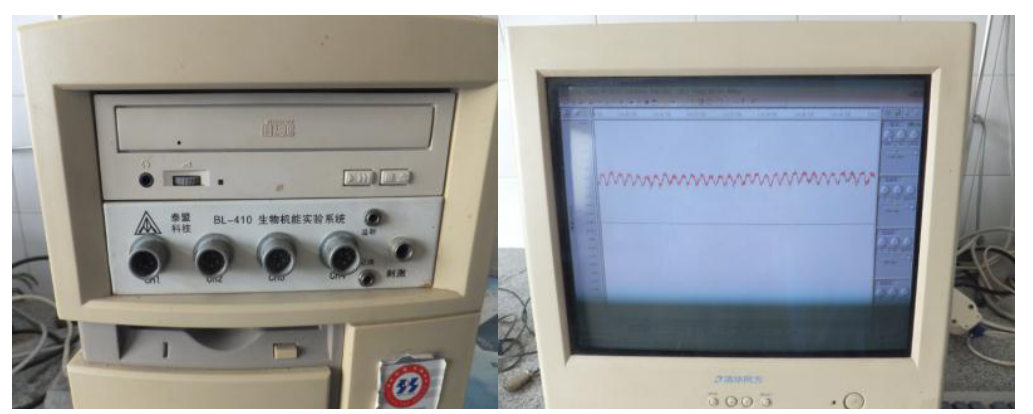

Fig. 2. BL-410 biological functional experiment system (on the left), and the blood vessels pulsation in the rabbits' vena cava displayed on the screen (on the right).

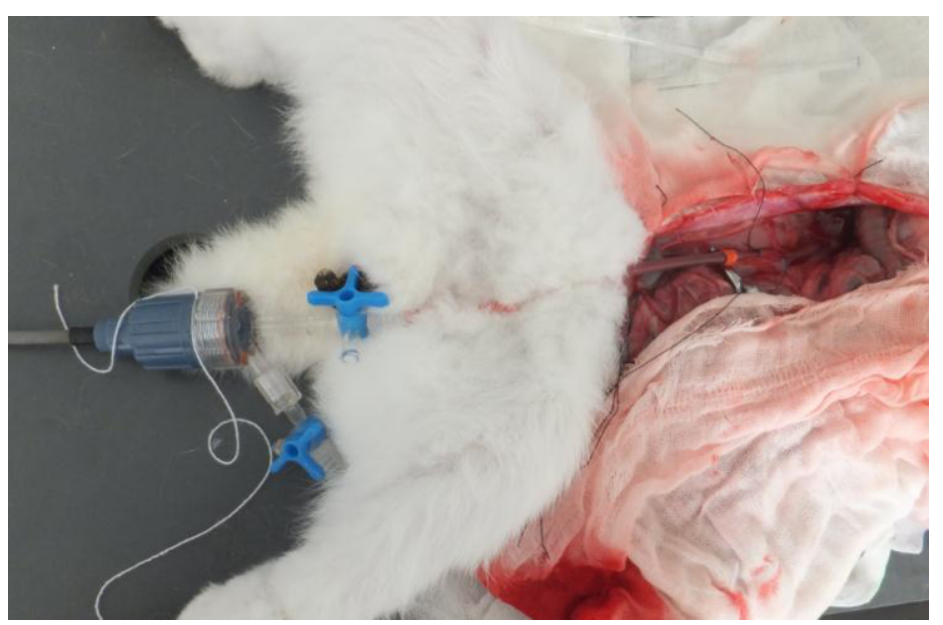

Fig. 3. Connection between the indwelling catheter for puncture and BL-410 Biological Functional Experiment System.

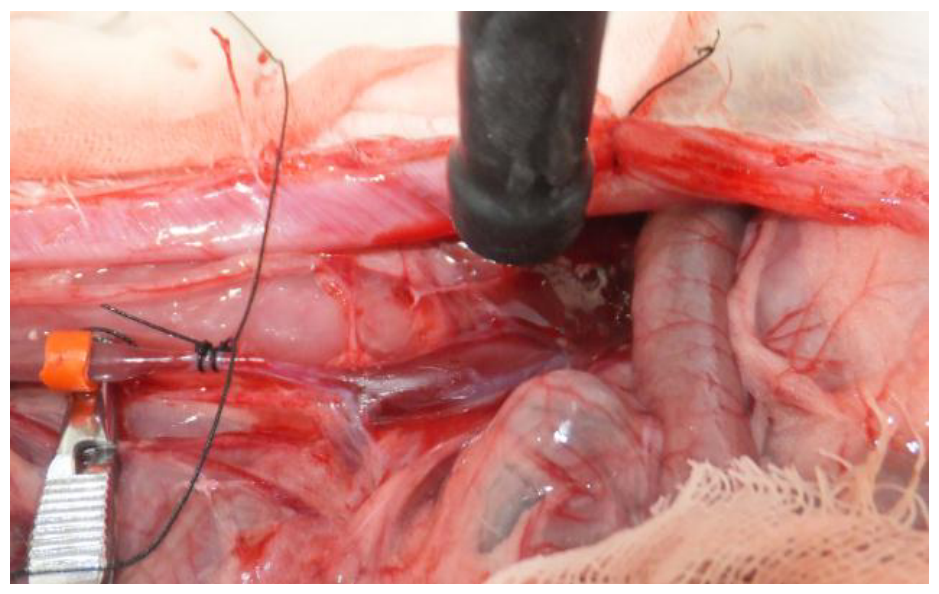

Fig. 4. Pressure measurement in the rabbit's inferior vena cava using the developed non-invasive measuring system.

above the exposed vena cava. As shown in Figure 4, the distance between the air outlet of the probe and the vascular wall was $10 \mathrm{~mm}$. Subsequently, we turned on the non-invasive measuring system. The pressure values in the vessel measured by this non-invasive measuring system and the needle 
puncture pressure values measured by BL-410 Biological Functional Experiment System were simultaneously recorded. By varying the tightness degree of the line knot close to the heart, the pressure values in the vessel measured by this non-invasive measuring system and the needle puncture pressure values measured by BL-410 Biological Functional Experiment System were also simultaneously recorded.

\subsection{Statistical analysis}

Data management and statistical analysis were performed using the IBM SPSS Statistics software (version 19), and the correlation between two variables were studied using line-regression analysis.

\section{Results}

The pressure measured by the BL-410 system and the non-invasive system were shown in Table 1 . The mean pressure of the BL-410 system and the non-invasive system were $702.1 \mathrm{~Pa}$ and $713.4 \mathrm{~Pa}$. There were no statistical differences between the two groups $(P>0.05)$. The linear regression equation: $\mathrm{Y}=1.001+10.820 \mathrm{X}(P<0.001), \mathrm{Y}$ is BL-410 system pressure, $\mathrm{X}$ is non-invasive system pressure. Two sets of data showed linear correlation.

\section{Discussions}

To make accurate predictions on esophageal variceal bleeding has always been an important research field in diagnosis and treatment of hepatic diseases. Many scholars all over the world have made a lot of attempts on this issue, and used a series of indexes to predict the probability of esophageal variceal bleeding, such as azygos vein blood flow (AVBF), portal venous pressure (PVP), esophageal variceal pressure (EVP), the hemodynamic ultrasound indexes of portal system, hepatic venous pressure gradient (HVPG), Liver stiffness and the endoscopic symptoms [15-17].

Table 1

Results measured by BL-410 biological functional experiment system and the developed non-invasive measuring system

\begin{tabular}{llllll}
\hline & BL-410 System $(\mathrm{Pa})$ & Non-invasive system $(\mathrm{Pa})$ & & BL-410 System $(\mathrm{Pa})$ & Non-invasive system $(\mathrm{Pa})$ \\
\hline 1 & 149.1 & 158.5 & 16 & 242.2 & 253.4 \\
2 & 347.2 & 358.4 & 17 & 473.7 & 485.4 \\
3 & 673.7 & 685.1 & 18 & 711.0 & 723.5 \\
4 & 1016.9 & 1029.6 & 19 & 908.1 & 917.4 \\
5 & 1140.5 & 1152.9 & 20 & 1129.4 & 1142.2 \\
6 & 309.9 & 321.8 & 21 & 231.4 & 244.1 \\
7 & 572.7 & 584.2 & 22 & 459.9 & 472.2 \\
8 & 808.1 & 817.5 & 23 & 702.2 & 713.5 \\
9 & 1040.5 & 1052.2 & 24 & 943.4 & 954.2 \\
10 & 1223.9 & 1232.5 & 25 & 1134.6 & 1145.5 \\
11 & 204.0 & 215.6 & 26 & 231.4 & 242.1 \\
12 & 448.2 & 457.2 & 27 & 458.9 & 467.5 \\
13 & 684.5 & 695.6 & 28 & 707.1 & 719.8 \\
14 & 925.7 & 29 & 933.6 & 945.6 \\
15 & 1105.2 & 937.3 & 30 & 1145.4 & 1157.2 \\
\hline
\end{tabular}


Unfortunately, the prediction accuracy with the use of these indexes is not very satisfactory. Currently, HVPG is generally regarded as the optimal index to diagnose esophageal varices and predict the bleeding by the scholars from various countries. However, the HVPG measurement should perform jugular venous cannula, i.e., the operation is invasive and sometimes dangerous. Additionally, the cost is high (in China, it costs approximately 6000 yuan for each examination), and the technology is also fairly difficult (it needs the assistance of interventional radiology team). As a consequence, the HVPG measurement technique now is far from being widely applied in clinical work. Moreover, HFPG reflects the pressure variation in hepatic sinusoid [18]. But the reason directly causing esophageal variceal bleeding is the pressure variation in esophageal veins, which cannot be accurately and timely reflected by the measurement of HVPG. So we can conclude that the selection of HVPG measurement as the gold standard in the prediction of esophageal variceal bleeding is actually a best of bad bunch at present, since the esophageal variceal pressure now is difficult to be measured safely, accurately and conveniently.

Currently, with regard to two kinds of non-invasive measurement methods, many in-vitro experiments, animal tests and clinical tests have been conducted. As reported, the results indicate that these two methods both exhibit clinical feasibility and significance $[19,20]$. The esophageal variceal pressure measurement results suggest that, when the esophageal variceal pressure is larger than 14 $\mathrm{mmHg}$, the bleeding rate exceeds $39 \%$; comparatively, when the esophageal variceal pressure is below $14 \mathrm{mmHg}$, only $9 \%$ of patients suffer from bleeding. Esophageal variceal pressure can directly reflect the hemodynamic conditions of esophageal veins, and azygos vein blood flow exhibits a positive correlation with vascular tension [21]. Using endoscopic balloon manometer, both in-vivo and in-vitro experimental results indicate that esophageal variceal pressure is highly correlated with HVPG and the other risk factors for bleeding. However, using endoscopic balloon manometer, the characteristic points in esophageal veins cannot be located and thus the pressure at these points cannot be accurately detected, i.e., the endoscopic balloon manometer also exhibits some limitations. When detecting the pressure of esophageal veins with comparatively large diameter, the vein puncture measurement method can be replaced by endoscopic balloon manometer. However, when detecting the pressure of the esophageal veins with comparatively small diameter, the balloon manometer has poor accuracy. The formation and development of esophageal varices is a gradual process. Therefore, for the esophageal veins with comparatively large diameter, the danger of bleeding is imminent and the quick treatment is urgently required. At that time, the measurement of esophageal variceal pressure is meaningless. In a conclusion, to predict esophageal variceal bleeding, we should find an endoscopic method with desirable safety, accuracy and repeatability, which can thus be used for measuring the pressure of esophageal veins with comparatively small diameter. Furthermore, using the esophageal variceal pressure as the core index and combining several auxiliary indices, we should develop a more comprehensive and reasonable prediction system for gaining in-depth insight into the physiopathologic mechanisms of the esophageal varices' formation and rupture bleeding.

With the esophageal varices' biological characteristics and many environmental factors in the esophagus taken into account, we use the pressure measurement in inferior vena cava in animals to simulate the pressure measurement in esophageal varices. The inferior vena cava is an important vein in blood circulation system. The circling heart blood flow from many visceral organs and tissues passes through the inferior vena cava, and accordingly, the blood flow volume passing through the inferior vena cava is great. In a living animal, we clipped the section of the inferior vena cava close to the heart. This blood circulation obstacle would lead to a rapidly rising pressure in inferior vena cava. Therefore, for a laboratory animal, we can quickly change the pressure in inferior vena cava by controlling its return. It's also worth mentioning that the diameter of inferior vena cava is generally 
great and the vascular wall is also thick compared with the other vessels, i.e., the inferior vena cava is comparatively strong and not easy to be damaged, which is conducive to performing the experiments with large pressure values. Additionally, in an animal, due to the effects of blood flow and visceral peristalsis, the inferior vena cava is not static and has some small activities, which is similar to varicose veins in peristaltic esophagus.

For the developed measuring system, accurate measurements of the airflow pressure on the surface of vascular wall and timely capture of the key moment when the vessel is flattened are two core problems. In previous studies, after preliminary debugging on the system, we also performed in-vitro simulation experiments and the experimental results suggested that the developed system could provide accurate measurements. After the airflow generated from the air pump passes through the pipeline with a constant length and inner diameter, the pressure at $1 \mathrm{~cm}$ away from the outlet exhibits a liner relationship with the pressure at the inlet. Based on the regression equation, the airflow pressure impacted on the examination parts can be quickly calculated according to the output pressure of air pump. In the present animal experiments, by measuring the pressure in the rabbit's inferior vena cava, we can observe that the results by the developed system fit well with the results by puncture measurement. Moreover, in non-static states, the developed system can also make accurate measurements, which will provide necessary experimental data and bases for further pressure measuring tests on humans.

In the developed system, the esophageal varices were impacted by the airflow with continuously varying pressures, and the deformation of esophageal varices can be assessed using image analyzing software. Then the pressure in vessels can be calculated based on the data of airflow impact force and deformation of vessels. Compared with the widely-adopted endoscopic balloon manometry, the present system exhibits a series of advantages as follows. Firstly, both the measurement method pressing against the vascular wall and balloon manometry will be in direct contact with the vessel inevitably. Considering that the vessels and the surrounding tissues are easily stimulated by the foreign matters, they may present irritable contraction after contact with the equipment, giving rise to measurement errors. Comparatively, the pressure measuring pipeline in the developed system is at a certain distance away from the veins, i.e., the developed system can offer a non-contact measurement in any real sense. Secondly, using the existing two methods, the effects of some interference factors such as esophageal peristalsis and contraction, cough, nausea and belching cannot be eliminated. Using the developed measuring system, the moments when the vessels are deformed can be quickly captured by the image analysis system in the manometer, and then the airflow pressure value at the instantaneous moment can be recorded. Even if small activities occur in the vessels, there will be little effects on the measurement results. Moreover, the operator can quickly find or even predict the variations which can affect the esophagus displacements in EV detection area in advance, and then effectively avoid the effects by turning off or turning on the airflow immediately. Since the proposed method can provide the results with excellent repeatability, it can perform multiple measurements in a short period and rapid multiple measurements when the vessel is return back to a static state. Using the proposed method, the measurement errors can be effectively avoided. Finally, the balloon pressure manometry includes a series of processes, mainly including balloon popping, air inflation to stick close to vascular wall, observing and pressure measuring, deflation, and thus is time-consuming. Using the developed system, the pressure values can be estimated according to the esophageal veins' morphological characteristics under gastroscope, and the airflow pressure value can be controlled to start from a close value instead of from zero, based on previous experience, i.e., the measuring time can be significantly shortened. More importantly, multiple measurements in one endoscopy operation can be realized. 


\section{Conclusion}

Therefore, it can be concluded that, the proposed non-invasive measuring system combining airflow and laser detection technology can be easily operated and provide the results with high accuracy. Moreover, it exhibits favorable feasibility and application prospects, which is expected to be improved in further human trials.

\section{Acknowledgment}

The study was supported by Hunan Provincial Natural Science Foundation of China (No. 12JJ4080) and the Fundamental Research Funds for the Central Universities of Central South University (No. 2011QNZT190 and No. 2012zzts034)

\section{References}

[1] R. de Franchis and Baveno V. Faculty, Revising consensus in portal hypertension: Report of the Baveno V. consensus workshop on methodology of diagnosis and therapy in portal hypertension, Journal of Hepatology 53 (2010), 762-768.

[2] G. Garcia-Tsao and J. Bosch, Management of varices and variceal hemorrhage in cirrhosis, The New England Journal of Medicine 362 (2010), 823-832.

[3] M. Soresi, L. Giannitrapani, M. Cervello, A. Licata and G. Montalto, Non-invasive tools for the diagnosis of liver cirrhosis, World Journal of Gastroenterology 20 (2014), 8131-8150.

[4] M. Poca, A. Puente, I. Graupera and C. Villanueva, Prognostic markers in patients with cirrhosis and portal hypertension who have not bled, Disease Markers 31 (2011), 147-154.

[5] J.L. Puckett, J. Liu, V. Bhalla, D. Kravetz, M.L. Krinsky, T. Hassanein and R.K. Mittal, Ultrasound system to measure esophageal varix pressure: An in vitro validation study, American Journal of Physiology-Gastrointestinal and Liver Physiology 288 (2005), G914-G919.

[6] G. Mehta, J.G. Abraldes and J. Bosch, Developments and controversies in the management of oesophageal and gastric varices, Gut 59 (2010), 701-705.

[7] R. de Franchis, Non-invasive (and minimally invasive) diagnosis of oesophageal varices, Journal of Hepatology 49 (2008), 520-527.

[8] R. Mosimann, Non-aggresive assessment of portal hypertension using endoscopic measurement of variceal pressure, American Journal of Surgery 143 (1982), 212-214.

[9] S.H. Zhu, X.Y. Liu, F.Z. Huang, W.P. Nei, B. Liu, R. Li, L.F. Cai, W. Wang, M. Yang and S.P. Ren, Calculation of esophageal variceal wall tension by ultrasonic microprobe and noninvasive pressure measurements, Romanian Journal of Gastroenterology 11 (2002), 9-11.

[10] P.H. Gertsch and J.J. Meister, Pressure measurement in oesophageal varices: Preliminary report on a new non-invasive method, Gut 28 (1987), 1162-1165.

[11] A.K. Vegesna, C.Y. Chung and A. Bajaj, Minimally invasive measurement of esophageal variceal pressure and wall tension (with video), Gastrointestinal Endoscopy 70 (2009), 414-416.

[12] L.S. Miller, Q. Dai, A. Thomas, C.Y. Chung, J. Park, S. Irizarry, T. Nguyen, V. Thangada, E.S. Miller and J.K. Kim, A new ultrasound-guided esophageal variceal pressure measuring device, American Journal of Gastroenterology 99 (2004), $1267-1273$.

[13] D.R. Kong, J.M. Xu, L. Zhang, C. Zhang, Z.Q. Fu, B.B. He, B. Sun and Y. Xie, Computerized endoscopic balloon manometry to detect esophageal variceal pressure, Endoscopy 41 (2009), 415-420.

[14] C.H. Hu, F.Z. Huang, R. Zhang, S.H. Zhu, W.P. Nie, X.Y. Liu, Y.L. Liu and P. Li, A new system for noninvasive esophageal varices pressure measurement based on airflow and laser technology, Chinese Journal of Medical lnstrumentation 38 (2014), 247-250.

[15] C. Zhang, D. Thabut, P.S. Kamath and V.H. Shah, Oesophageal varices in cirrhotic patients: From variceal screening to primary prophylaxis of the first oesophageal variceal bleeding, Liver International 31 (2011), 108-119.

[16] C. Luigiano, G. Iabichino, A. Judica, C. Virgilio, V. Peta and L. Abenavoli, Role of endoscopy in management of gastrointestinal complications of portal hypertension, World Journal of Gastrointestinal Endoscopy 7 (2015), 1-12. 
[17] A. Berzigotti, R. Gilabert, J.G. Abraldes, C. Nicolau, C. Bru, J. Bosch and J.C. García-Pagan, Noninvasive prediction of clinically significant portal hypertension and esophageal varices in patients with compensated liver cirrhosis, The American Journal of Gastroenterology 103 (2008), 1159-1167.

[18] J.G. Abraldes, C. Villanueva, R. Bañares, C. Aracil, M.V. Catalina, J.C. Garcia-Pagán and J. Bosch, Spanish cooperative group for portal hypertension and variceal bleeding, hepatic venous pressure gradient and prognosis in patients with acute variceal bleeding treated with pharmacologic and endoscopic therapy, Journal of Hepatology 48 (2008), 229-236.

[19] K.A. Brensing, M. Neubrand, J. Textor, P. Raab, H. Müller-Miny, C. Scheurlen, J. Görich, H. Schild and T. Sauerbruch, Endoscopic manometry of esophageal varices: Evaluation of an endoscopic balloon technique compared with direct portal pressure measurement, Journal of Hepatology 29 (1998), 94-102.

[20] L. Spahr, E. Giostra, I. Morard, G. Mentha and A. Hadengue, Perendoscopic variceal pressure measurement: A reliable estimation of portal pressure in patients with cirrhosis? Gastroentérologie Clinique et Biologique 30 (2006), 1012-1018.

[21] C. Scheurlen, A. Roleff, M. Neubrand and T. Sauerbruch, Noninvasive endoscopic determination of intravariceal pressure in patients with portal hypertension: Clinical experience with a new balloon technique, Endoscopy 30 (1998), 326-332. 